\title{
CANCELING BRANCH POINTS ON PROJECTIONS OF SURFACES IN 4-SPACE
}

\author{
J. SCOTT CARTER AND MASAHICO SAITO
}

(Communicated by Frederick R. Cohen)

\begin{abstract}
A surface embedded in 4-space projects to a generic map in 3-space that may have branch points-each contributing \pm 1 to the normal Euler class of the surface. The sign depends on crossing information near the branch point. A pair of oppositely signed branch points are geometrically canceled by an isotopy of the surface in 4-space. In particular, any orientable manifold is isotopic to one that projects without branch points. This last result was originally obtained by Giller. Our methods apply to give a proof of Whitney's theorem.
\end{abstract}

\section{INTRODUCTION}

In the sequel all maps and manifolds are standardly smooth and in general position. Such maps of surfaces in 3-space have isolated branch points; examples of these are illustrated in Figure A on the next page.

Giller [6] proved that any orientable embedded surface in $\mathbf{R}^{4}$ can be isotoped so that the projection onto $\mathbf{R}^{3}$ is an immersion. Roseman [8] generalized this to arbitrary high dimensional codimension 2 embeddings and gave a different proof that handled some nonorientable manifolds with trivial normal Euler class. This integer class is, as Giller observed, an obstruction to lifting an immersion in $\mathbf{R}^{3}$ of the projective plane to an embedding in $\mathbf{R}^{4}$.

We prove the following

Theorem 1. Let $F$ be an embedded surface in $\mathbf{R}^{4}$, with normal Euler number $e$. Then there is an isotopic embedding of $F$ that projects in $\mathbf{R}^{3}$ to a map with $|e|$ branch points. This is the minimum number of branch points that can be achieved among all isotopic embeddings.

Corollary 2. The embedded surface $F$ can be isotoped so that the projection is an immersion if and only if $e=0$.

Our proof of Theorem 1 is in the same spirit as Roseman's proof of similar results [8]; here is a sketch. Each branch point contributes \pm 1 to the normal

\footnotetext{
Received by the editors November 20, 1990; presented at AMS 861, Denton, Texas Nov. 2-3, 1990.

1991 Mathematics Subject Classification. Primary 57Q35; Secondary 57Q45.

Key words and phrases. Embedded surfaces in 4-space, projections, branch points, normal Euler number.

The first author was supported by a grant from the University of South Alabama Research Committee.
} 

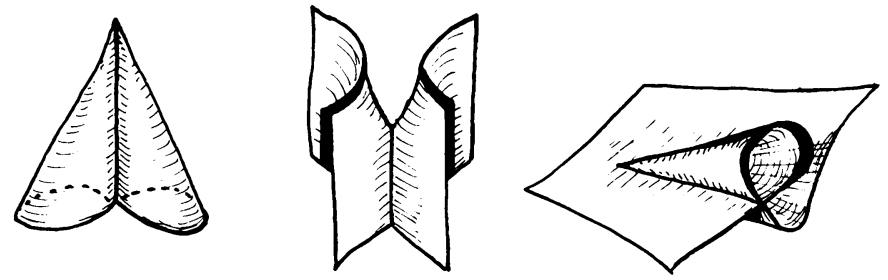

Figure A. Branch points

Euler number according to the local crossing information near the branch point. Thus branch points fall into two classes: positive and negative. Then each pair of positive and negative branch points are canceled by isotopy. Thus a minimum number is achieved. The isotopies are chosen to involve only two of Roseman's Reidemeister type moves [9]. The details of the proof are presented in $\S \S 2$ and 4.

At the expense of adding genus, each triple point of the projected surface $p(F)$ can be replaced with a pair of branch points. This trick gives an alternative proof of Whitney's Theorem that we present in $\S 5$.

Theorem 3 (Whitney [11]). The normal Euler number, $e$, of an embedding of $a$ surface $F$ in 4-space satisfies the congruence, $\frac{1}{2} e \equiv \chi(\bmod 2)$, where $\chi$ denotes the Euler characteristic of the surface.

In $[1,2,3]$, Tom Banchoff gave the formulation of the normal Euler class in terms of the indices of the branch points and proved Lemma 4 in the piecewiselinear category. Upcoming work of Banchoff and Johnson [4] contains more on the PL theory. In the main theorem of [10], Bruce Trace gave a proof of a relative version of Theorem 1 using similar techniques. We remark that there is a strong interplay among triple points, branch points, and knottings of surfaces that wants further exploration.

\section{SigNS OF BRANCH POINTS}

Let $F$ be an embedded surface in $\mathbf{R}^{4}$, and let $p: \mathbf{R}^{4} \rightarrow \mathbf{R}^{3}$ be a generic projection. The normal Euler number can be calculated as follows. Let $F_{1}$ be a transverse push-off of $F$. At each point of $F \cap F_{1}$, choose a local orientation of $F$ and an induced orientation of $F_{1}$. The local intersection number between these surfaces is computed by comparing the orientation of the two intersecting surfaces with a fixed orientation of $\mathbf{R}^{4}$. The normal Euler class $e(F)$ is the sum of these intersection numbers taken over all the points of $F \cap F_{1}$.

The boundary of a regular neighborhood of an intersection point inherits an orientation from $\mathbf{R}^{4}$. In this 3 -sphere, the surfaces $F$ and $F_{1}$ form an oriented Hopf link (with orientation induced from the local choices). The local intersection number is the linking number as computed in this oriented 3-sphere.

A sufficiently small ball neighborhood, $B$, of a branch point on $p(F)$ in $\mathbf{R}^{3}$ intersects $p(F)$ in the cone on a figure eight; thus $p(F) \cap \partial B$ is a figure eight in $S^{2}$. Consider a disk, $D$, in $\partial B$ that contains the intersection $p(F) \cap \partial B$. See Figure B. 


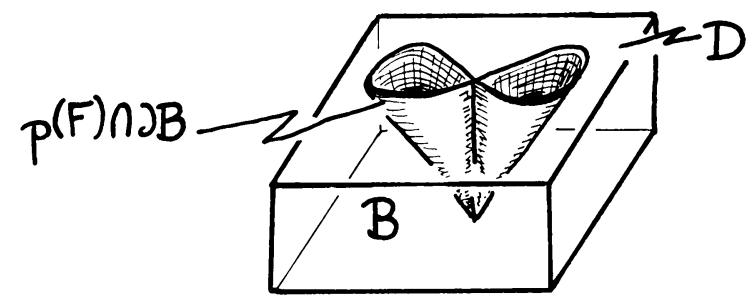

Figure B. A neighborhood of a branch point
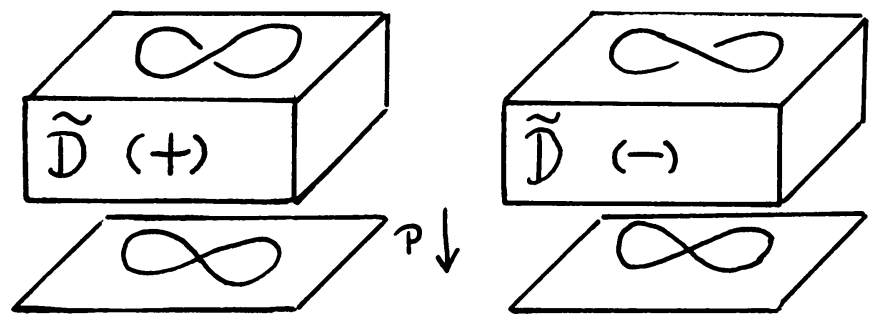

Figure C. Lifts of the figure 8

A 3-ball, $\widetilde{D}$, contained in $\mathbf{R}^{4}$ that projects to $D$ can be chosen small enough so that $F \cap \widetilde{D}$ looks like either Figure $\mathrm{C}(+)$ or $\mathrm{C}(-)$ where these figures have right-handed coordinate systems. The branch point is called positive or negative if the intersection $F \cap \widetilde{D}$ resembles Figure $\mathrm{C}(+)$ or $\mathrm{C}(-)$, respectively.

Hence a branch point is positive if, in a movie of the surface, it is born with negative writhe or dies with positive writhe. The difference in signs for birth and death occurs because the orientation of the disk $\widetilde{D}$ (that is induced from $\mathbf{R}^{4}$ ) is left-handed if $\widetilde{D}$ appears at the bottom of a movie.

Lemma 4 (Banchoff). The number of positive branch points minus the number of negative branch points is equal to the normal Euler number.

Proof. Let $b_{1}, \ldots, b_{n}$ be the branch points on $p(F)$. Let $B_{i}, D_{i}, \widetilde{D}_{i}$ be the corresponding neighborhoods and disks described above $(i=1, \ldots, n)$. Let $\widetilde{B}_{i}$ be a small 4-ball neighborhood of $p^{-1}\left(b_{i}\right)$ such that $p\left(\widetilde{B}_{i}\right)=B_{i}$ and $\widetilde{D}_{i} \subset \widetilde{B}_{i}$. Set $F^{\prime}=F \backslash \bigcup \operatorname{Int}\left(\widetilde{B}_{i} \cap F\right)$. The complement of the singularities, $F^{\prime}$, is properly embedded in $\mathbf{R}^{4} \backslash \cup \operatorname{Int}\left(\widetilde{B}_{i}\right)$.

Push $F^{\prime}$ slightly in the projectional direction in $\mathbf{R}^{4}$; the push-off $F^{\prime \prime}$ can be made to be properly embedded in $\mathbf{R}^{4} \backslash \bigcup \operatorname{Int}\left(\widetilde{B}_{i}\right)$. Since $\left.p\right|_{F^{\prime}}$ is an immersion, the push is nontangential and $F^{\prime} \cap F^{\prime \prime}$ is empty. Now $F^{\prime \prime} \cap \widetilde{D}_{i}$ looks like Figure $\mathrm{D}$ if $b_{i}$ is a positive branch point in a right-handed ball. If $b_{i}$ is negative, the crossing information is opposite or the orientation induced in $\widetilde{D}$ is left-handed.

Give compatible local orientations to $F^{\prime}$ and $F^{\prime \prime}$ in $\widetilde{B}_{i}$. Then Figure D shows that the linking number between $F^{\prime} \cap \widetilde{B}_{i}$ and $F^{\prime \prime} \cap \widetilde{B}_{i}$ in $\partial \widetilde{B}_{i}\left(\cong S^{3}\right)$ is 1 (or $(-1)$ if $b_{i}$ is negative). The lemma follows by taking summation of these linking numbers over all branch points. 


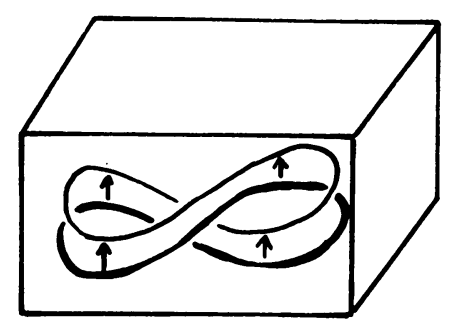

Figure D. A translation of figure 8

\section{REMARK}

Cappell and Shaneson [5] studied the relationship between characteristic classes (of the tangent bundle) and the singularities of maps from manifolds to Euclidean spaces. Thus there are relationships between normal characteristic classes and singularities of projections via Whitney's product formula. Lemma 4 suggests that there are more direct and stronger relationships between them. More precisely, it would be conjectured that the singularities of projections together with the local crossing information are dual to the normal characteristic classes of the embeddings.

\section{Canceling branch POINTS}

Lemma 5. If there is a pair of positive and negative branch points on $p(F)$, then the number of branch points can be reduced by 2 via an ambient isotopy of $F$ in $\mathbf{R}^{4}$.

The idea of the proof of this lemma is similar to Roseman's pig-tail trick [8].

Let $b_{+}$and $b_{-}$be a pair of positive and negative branch points and set $\tilde{b}_{ \pm}=p^{-1}\left(b_{ \pm}\right)$. Let

$$
\Sigma=\operatorname{Closure}(\{x \in F \mid p(x)=p(y) \text { for some } y \neq x\}) .
$$

Then $\Sigma$ is a collection of immersed circles in $F$ and $\tilde{b}_{ \pm} \in \Sigma$.

Choose an embedded arc $\gamma$ on $F$ connecting $\tilde{b}_{+}$to $\tilde{b}_{-}$such that $\gamma$ is perpendicular to $\Sigma$ at its ends as depicted in Figure E. The projection appears as Figure F. The interior of the arc $\gamma$ is to intersect $\Sigma$ transversely; thus $\gamma$ does not pass through triple points upon projection, and its image $p(\gamma[0,1])$ is an embedding. It is possible that $\gamma$ crosses an arc in $\Sigma$ as in Figure $\mathbf{H}$.

Pull the branch point $b_{+}$along $p(\gamma)$ towards $b_{-}$as indicated in Figure G. This move is realized by an ambient isotopy of $F$ in $\mathbf{R}^{4}$.

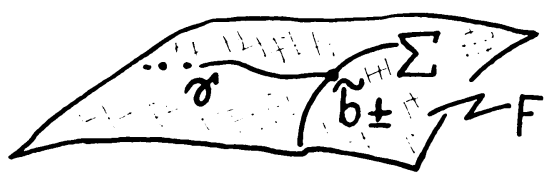

Figure E. The end of a connecting arc 


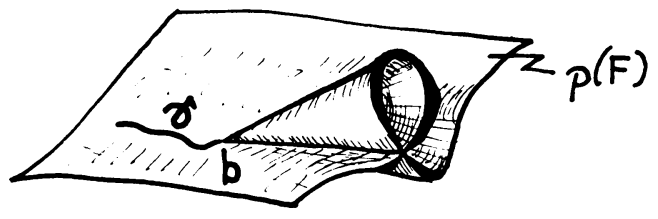

Figure F. A projection of an arc near the branch point

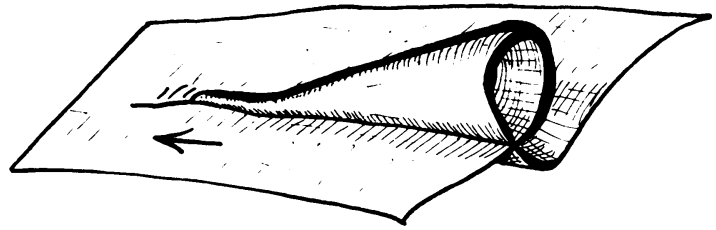

FIGURE G. Moving a branch point along the arc
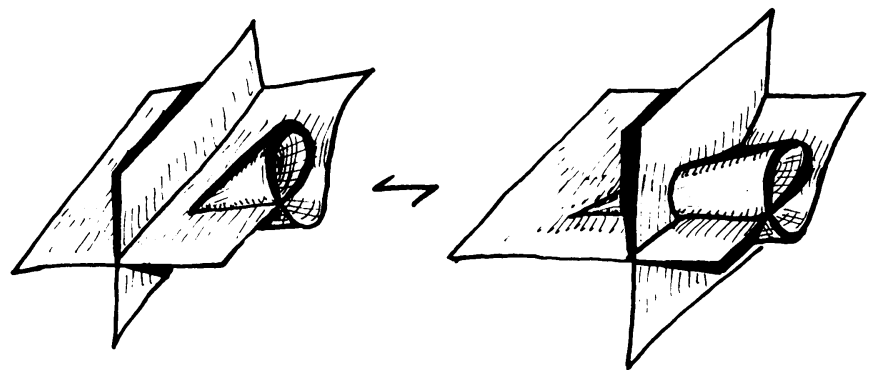

FIGURE H. Passing through the sheet

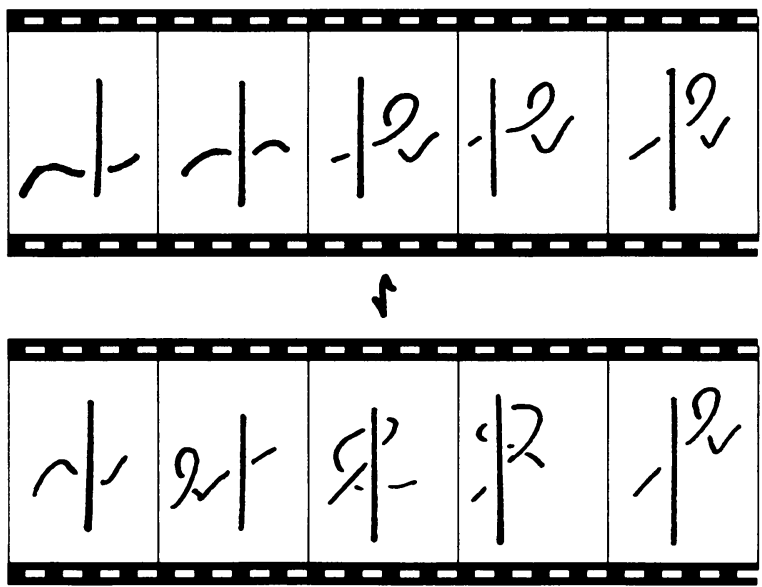

Figure I. Passing through the sheet: The movie

If $p(\gamma)$ passes through $p(F)$ transversely as indicated in Figure $\mathrm{H}$, then the branch point will pass through the sheet, and a triple point is introduced. Figure $\mathrm{H}$ depicts the projection of this move; a movie appears in Figure I. The move is realized by an ambient isotopy, and Roseman [9] considers this as an elementary Reidemeister type move. 


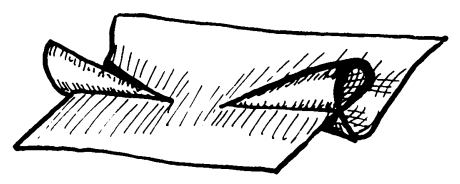

Figure J.

Branch points on the same side

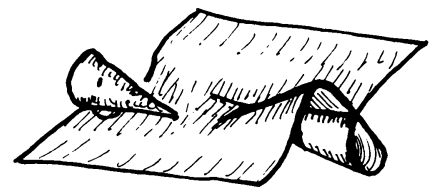

FIGURE K.

Branch points on the opposite sides

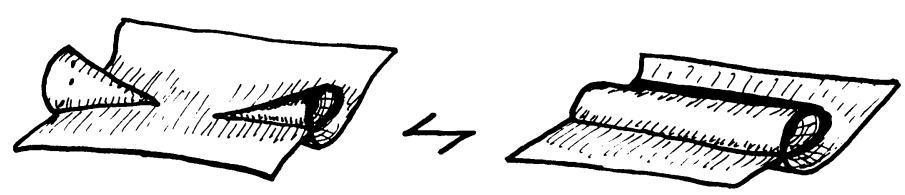

Figure L. A Roseman's move
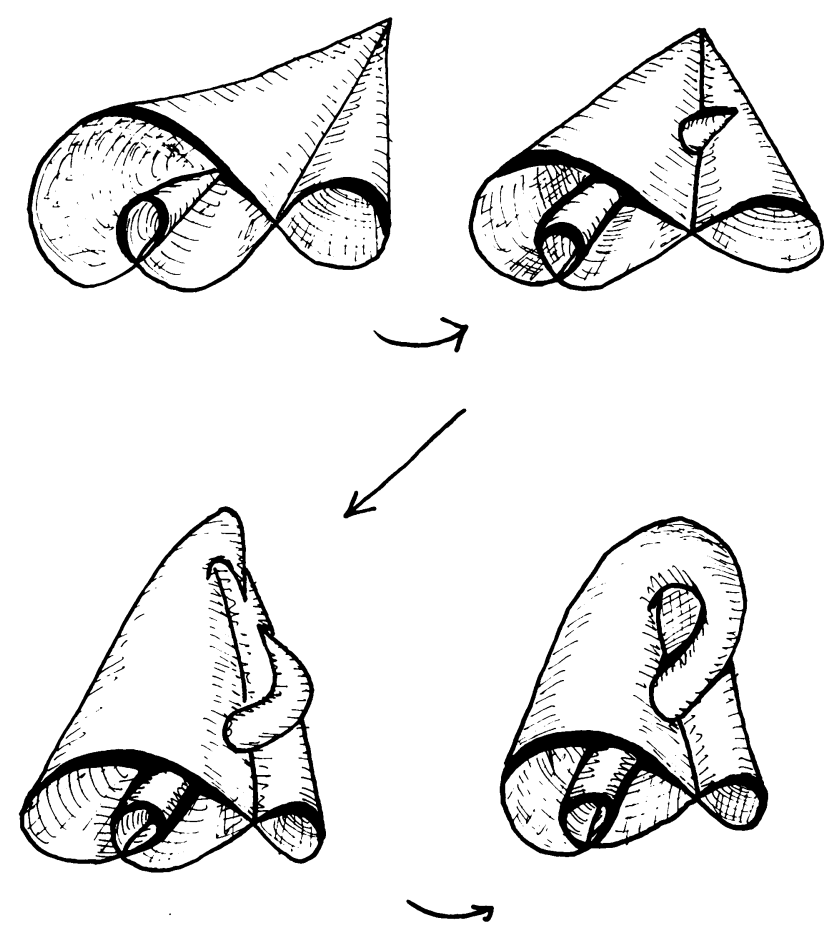

FIGURE M. An isotopy between kinky disks

Thus the oppositely signed branch points $b_{+}$and $b_{-}$can be brought together so they lie in a common neighborhood. In such a neighborhood the projection looks like one of the two cases illustrated in Figure $\mathbf{J}$ or Figure $\mathrm{K}$. In the case of Figure J, one of the Roseman's moves cancels $b_{+}$with $b_{-}$as indicated in Figure L. In the case of Figure $K$, an isotopy shown in Figure $M$ will cancel them. In either of these scenerios, the points have opposite signs, hence they have the 
same writhe, and hence the moves are realizable (cf. Roseman's remarks [9] on the meaning of his moves). The result follows.

Theorem 1 follows from the above lemmas since pairs of oppositely signed branch points can be canceled successively.

\section{A PROOF OF WHITNEY'S THEOREM}

Kamada [7] gave elementary proofs of Whitney's and Massey's theorems. Here we present a proof of Whitney's theorem using projections. Our proof is similar to Kamada's in that handles are attached to simplify the map, but the point here is the extensive use of projections.

Proof of Whitney's theorem. Let an embedding be given, and consider the projection $p(F)$. Triple points of $p$ can be removed as follows. Consider any generic projection of the given embedded surface $F$. Each triple point in the projection corresponds to a type III Reidemeister move in a movie of the embedding. Figure $\mathrm{N}$ shows how to replace the triple point with a pair of branch points of opposite sign. Figure $\mathrm{O}$ on the next page depicts the movie of
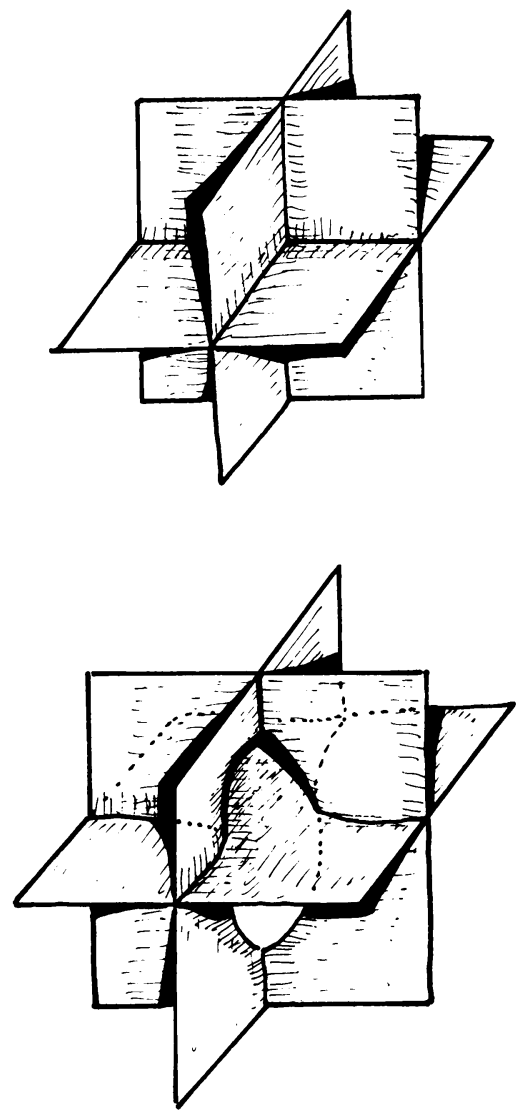

FIGURE N. Removing triple points 

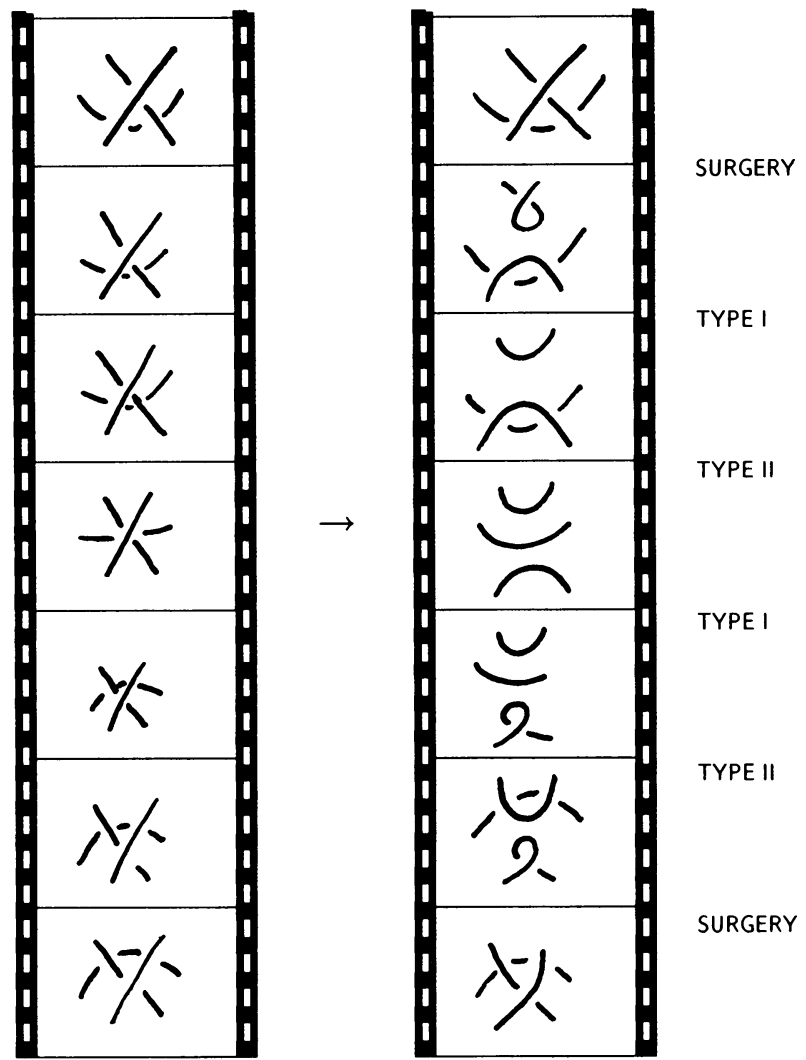

FIGURE O. Removing triple points: the movie

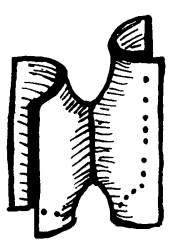

Figure P. An a-arc

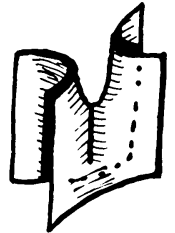

FIGURE Q. An m-arc

this trick. In these figures, a hollow 1-handle has been added to $F$, an arc of double points has been removed, and a pair of oppositely signed branch points has been introduced.

Notice that a similar trick can be performed in the absence of a triple point: Ignore the third sheet in Figure O or Figure N.

Therefore, by performing the trick at all the triple points of $p(F)$, the projection may be assumed to have no triple points. In this case, each double point curve is embedded in $\mathbf{R}^{3}$ as a simple closed curve, or as an arc that ends in branch points. For any given simple closed loop of double points, the trick performed at a point in the loop replaces the loop with an arc. 
In this way, an embedded surface $G$ in $\mathbf{R}^{4}$ is found such that (1) the normal Euler class of $G$ coincides with the normal class of $F,(2)$ the characteristic of $G$ has the same parity as does the characteristic of $F$, and (3) the double point set of $p(G)$ consists of embedded arcs.

There are two types of arcs. An arc is called an $a$-arc (resp. $m$-arc) if the lift of the neighborhood of the arc is an annulus (resp. a Mobius band). (See Figures $\mathrm{P}$ and $\mathrm{Q}$.)

Since $p\left(F_{1}\right)$ is the projection of an embedding, the signs of the ends of an a-arc differ, but the signs of the ends of an m-arc coincide.

Remove the neighborhoods of arcs and attach disks along boundary to get an embedded surface $G_{1}$ in $\mathbf{R}^{4}$ that projects to an embedding in $\mathbf{R}^{3}$. In the case of an a-arc the replacement does not change $\chi$ modulo 2 and $\frac{1}{2} e$ modulo 2 . For an m-arc, both $\frac{1}{2} e$ and $\chi$ change parity. The theorem follows since the congruence is certainly true for embedded surfaces in $\mathbf{R}^{3}$.

\section{ACKNOWLEDGMENTS}

The authors are grateful to Professors Cameron McA. Gordon and Dan Silver for valuable conversations.

\section{REFERENCES}

1. T. F. Banchoff, Double tangency theorems for pairs of submanifolds, Geometry Symposium Utrecht 1980 (Looijenga, Seirsma, and Takens, eds.), Lecture Notes in Math., vol. 894, Springer-Verlag, Berlin and New York, 1981, pp. 26-48.

2. _ Normal curvature and Euler classes for polyhedral surfaces in 4-space, Proc. Amer. Math. Soc. 92 (1984), 593-596.

3. _ The normal Euler class of a polyhedral surface in 4-space, unpublished notes and letters.

4. T. F. Banchoff and Ockle Johnson (to appear).

5. S. E. Cappell and J. L. Shaneson, An introduction to embeddings, immersions and singularities in codimension two, Proc. Sympos. Pure Math., vol. 32, Amer. Math. Soc., Providence, RI, 1978.

6. Cole Giller, Towards a classical knot theory for surfaces in $\mathbf{R}^{4}$, Illinois J. Math. 26 (1982), 591-631.

7. Seiichi Kamada, Non-orientable surfaces in 4-space, Osaka J. Math. 26 (1989) 367-385.

8. Dennis Roseman, Projections of codimension two embeddings, preprint.

9. __ Reidemeister-type moves for surfaces in four dimensional space, preprint.

10. Bruce Trace, A general postion theorem for surfaces in 4-space, Comtemp. Math., vol. 44, Amer. Math. Soc., Providence, RI, 1985, pp. 123-137.

11. H. Whitney, On the topology of differentiable manifolds, Lectures in Topology (Raymond L. Wilder and William L. Ayres, eds.), Univ. of Michigan Press, Ann Arbor, 1941.

Department of Mathematics and Statistics, University of South Alabama, Mobile, ALABAMA 36688

E-mail address: F4T3@USOUTHAL.BITNET

Department of Mathematics, University of Texas at Austin, Austin, Texas 78712

E-mail address: saito@math.utexas.edu 